\title{
A Modeling Study of Nerve Fascicle Stimulation
}

\author{
PETER H. VELTINK, MEMBER, IEEE, BENNO K. VAN VEEN, JOHANNES J. STRUIJK, \\ JAN HOLSHEIMER, AND HERMAN B. K. BOOM
}

\begin{abstract}
A nerve stimulation model has been developed, incorporating realistic cross-sectional nerve geometries and conductivities. The potential field in the volume conductor was calculated numerically using the variational method. Nerve fiber excitation was described by the model of McNeal.

Cross-sectional geometries of small monofascicular rat common peroneal nerve and multifascicular human deep peroneal nerve were taken as sample geometries. Selective stimulation of a fascicle was theoretically analyzed for several electrode positions: outside the nerve, in the connective tissue of the nerve, and inside a fascicle. The model results predict that the use of intraneural or even intrafascicular electrodes is necessary for selective stimulation of fascicles not lying at the surface of the nerve.

Model predictions corresponded with experimental results of Veltink $e t$ al. on intrafascicular and extraneural stimulation of rat common peroneal nerve and to results of McNeal and Bowman on muscle selective stimulation in multifascicular dog sciatic nerve using an extraneural multielectrode configuration.
\end{abstract}

\section{INTRODUCTION}

A RTIFICIAL nerve stimulation as a method for the rehabilitation of spinal cord injured patients has been of growing interest during the last years. It is generally believed that its functional performance will improve if more physiological contraction patterns can be realized than is currently possible with cuff electrodes with only one electrode or pairs of electrodes [1]-[9]. One approach is the use of multielectrode configurations for the independent control of several groups of motoneuron fibers within the nerve [1], [2], [6], [8], [9]. A prerequisite for the advantageous application of this approach is a limited overlap of motoneuron groups recruited by different electrodes. This means that only few motoneurons are excited by more than one electrode.

Multielectrode configurations can be used for the alternating stimulation of groups of motor units belonging to a single muscle or group of synergistic muscles. This method can reduce muscle fatigue which occurs if the same motor unit group always is synchronously activated by only one electrode. Holle et al. [1] observed an improved resistance to muscle fatigue when motoneuron groups in the phrenic nerve of dogs were activated in turn by a multielectrode configuration. The same was observed by Moritz et al. [10] who stimulated phrenic nerves of patients. By stimulating alternately electrodes at dif-

Manuscript received April 25, 1988; revised October 28, 1988.

The authors are with the Biomedical Engineering Division, Department of Electrical Engineering, University of Twente, 7500 Enschede, The Netherlands.

IEEE Log Number 8927447 ferent sides of the sciatic nerve of cat, Petrofsky [2] found a similar fatigue resistance as when stimulating the three split parts of the associated ventral roots in turn. However, he did not compare these results with fatigue resistance obtained when stimulating with only one electrode.

Another possibility created by the use of multielectrode configurations for nerve stimulation is the independent control of groups of motor neuron fibers belonging to muscles or muscle groups with different functions. In this situation, the motoneuron groups recruited by different electrodes should not only have a limited overlap, but should also belong to different muscles or muscle groups. McNeal and Bowman [6] investigated selective stimulation of four muscles of the hindlimb of dogs when stimulating with a seven-electrode cuff configuration around the sciatic nerve before its bifurcation into tibial and peroneal nerves. At this level, tibial and peroneal nerve fibers lie in fascicles at different sides of the nerve. By bipolar stimulation between electrodes at the same side of the nerve, extensor, and flexor muscles could be stimulated selectively.

The performance of multielectrode configurations could be improved, if it would be possible to selectively stimulate a larger number of smaller groups of motoneuron fibers, not only close to the surface of the nerve, but also deeper inside. Limitations regarding the size of these groups were investigated by Veltink et al. [8], [9]. They considered the overlap of motoneuron fibers recruited within the single fascicle of rat common peroneal nerve when stimulating intrafascicularly or extraneurally with a multielectrode in one cross section of the nerve. An appreciable overlap of recruited groups of motoneuron fibers was found. Selective stimulation of a single muscle could not be obtained and no appreciable difference of muscle specificity among motoneuron groups recruited by different electrodes was found. Thus, by stimulating a single fascicle with a multielectrode in this way, the results were not as intended.

In nerves with many fascicles, groups of motoneuron fibers are distributed among fascicles. Because these fascicles have different positions in the nerve, they may be stimulated selectively by electrodes in their neighborhood. Furthermore, selective stimulation of fascicles might result in selective stimulation of muscles, if the fascicles have different muscle specificities. Differences of muscle specificity among fascicles depend on the position along the nerve [11]. Selective stimulation of fascicles within a multifascicle nerve has not yet been investigated 
experimentally. In this paper, the results of a theoretical analysis on this subject are presented. Simulations were performed with a realistic geometrical model of multifascicular human deep peroneal nerve and monofascicular rat common peroneal nerve.

\section{Methods}

\section{Computation of the Electrical Potential Distribution}

The potential distribution in a realistic geometrical nerve model was calculated numerically with the variational method [12], [13] under quasi-stationary conditions. The medium was assumed to be purely resistive. The nerve volume was divided into identical wedgeshaped volume elements of thickness $k$ (Fig. 1). The geometry of each wedge in the $x y$ plane perpendicular to the longitudinal axis ( $z$ dimension) is an equilateral triangle with sides $h$. The nerve model consists of 40 layers of volume elements along the nerve ( $z$ direction). Each layer consists of 40 rows of from 61 to 99 volume elements in the $x$ direction. A dissipation functional $D(\varphi)$, defined on the volume $V$ of the nerve model

$$
\begin{aligned}
D(\varphi)= & \iiint_{V} \frac{1}{2}\left\{\sigma_{x}\left(\frac{\partial \varphi}{\partial x}\right)^{2}+\sigma_{y}\left(\frac{\partial \varphi}{\partial y}\right)^{2}\right. \\
& \left.+\sigma_{z}\left(\frac{\partial \varphi}{\partial z}\right)^{2}\right\} d V
\end{aligned}
$$

was discretized as the sum $\Sigma D_{e}(\varphi)$ of contributions from all elements and the dissipation $D_{e}(\varphi)$ in an element was approximated by a quadratic expression in the potentials at the six corner points (grid points) (see Appendix B). Straightforward minimization of the discretized total dissipation leads to a linear system of algebraic equations for the unknown potential values at the grid points.

The iterative numerical method used for solving these equations reflects the point/row/plane hierarchy. The electrical potentials of the grid points of one single row were computed simultaneously by Gaussian elimination and backward substitution applied on a tridiagonal matrix [14], while the potentials at grid points of neighboring rows were taken constant. The Gauss-Seidel method [14] was used for iteration on the rows of a single plane (plane iteration). Furthermore, iterations on all 40 planes of the model were performed using the Gauss-Seidel method in combination with successive over-relaxation [14]. The over-relaxation factor used was 1.7. One full model iteration consisted of eight plane iterations for each of the 40 layers. Full model iterations were performed until the following criterion was met:

$$
\frac{\sum_{i=1}^{n}\left|\varphi_{i}^{j+1}-\varphi_{i}^{j}\right|}{\sum_{i=1}^{n}\left|\varphi_{i}^{j}\right|}<0.003
$$

with $\varphi_{i}^{j}$ being the potential at grid point $i$ after iteration number $j$, and $n$ the total number of grid points.

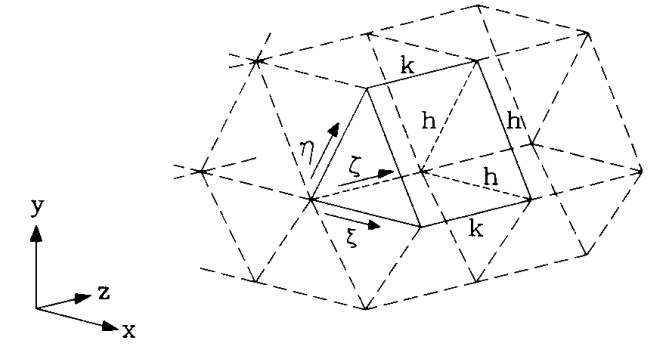

Fig. 1. Composition of the three-dimensional model by wedge shaped volume elements of height $k$ in the direction of the nerve axis ( $z$ direction), and the shape of equilateral triangles with sides $h$ in the $x y$ plane. An alternative coordinate system $\xi \eta \zeta$ was used for each volume element with $\xi, \eta$, and $\zeta$ directions defined along the sides of the volume element.

In the nerve model, a single stimulating electrode was incorporated. We assumed that nerve geometry did not change within twice the model length. Also, the $x y$ plane in which the electrode was located, was assumed to be a plane of symmetry in the model. Therefore, only half of the nerve length had to be modeled and the boundary condition for the symmetry plane was a Neumann condition: zero current density normal to this surface was assumed. The boundary condition for the rest of the model surface was of the Dirichlet type: potential was set to zero. For computational reasons, the electrode itself was also modeled as a Dirichlet condition (imposed potential). However, the results are comparable to injecting current at the electrode surface because the tissue was assumed to be purely resistive.

\section{Cross-Sectional Geometry of Modeled Nerves}

In this study two types of nerve were considered: the common peroneal (CP) nerve of rat, with only one main fascicle, and the human deep peroneal (DP) nerve, made up of many fascicles. The cross-sectional geometry of the CP nerve [Fig. 2(a)] was obtained from material used in stimulation experiments [8], [9]. After an experiment the CP nerve was removed and part of it was frozen and cut into slices. The slices were treated with Sudan Black, which selectively stains the myelin sheath of the nerve fibers. The sides of the elementary triangles in the cross section of the model of this nerve [Fig. 2(b)] are $h=0.02$ $\mathrm{mm}$. The rat $\mathrm{CP}$ at this level mainly constitutes of one fascicle with a diameter of approximately $0.5 \mathrm{~mm}$.

The human DP nerve was chosen as an example of a nerve with many fascicles [Fig. 3(a)], each containing motoneuron fibers for a specific muscle or part of a muscle [11]. The geometrical nerve model constructed from a cross section of this nerve is shown in Fig. 3(b). In this model, the sides of the elementary triangles are $h=0.09$ $\mathrm{mm}$. Five fascicles were given a code for their identification. The diameter of the nerve at this level is approximately $3.8 \mathrm{~mm}$ [11], [15].

\section{Electrical Conductivities}

The nerve models used in this study consist of a number of compartments with different conductivities, summarized in Table $I$. 


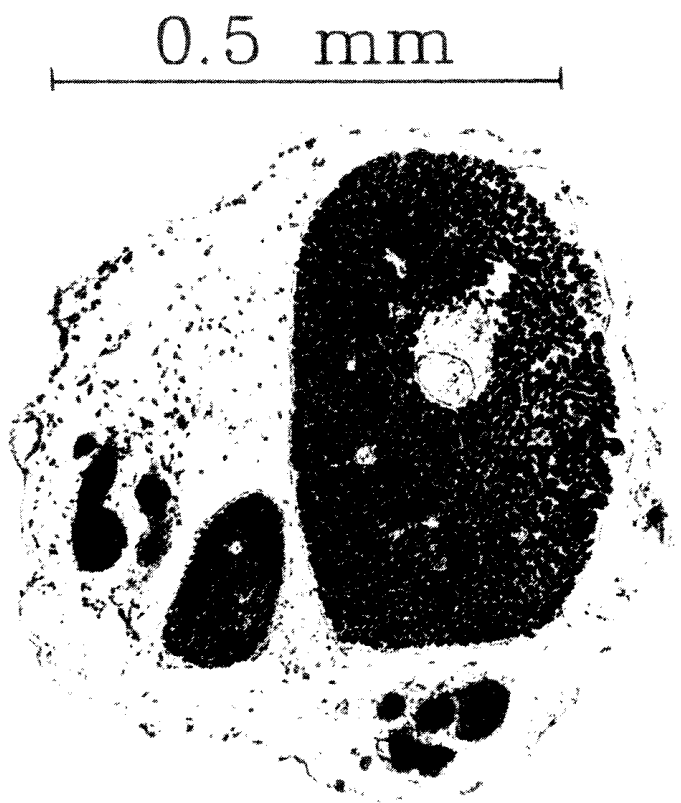

(a)

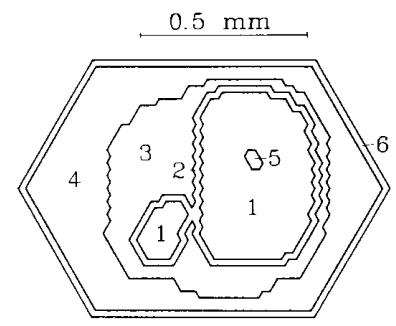

(b)

Fig. 2. Rat common peroneal (CP) nerve. (a) Photograph of a cross-sectional slice, from which the nerve model geometry was determined. (b) Cross section of nerve model $(h=0.02 \mathrm{~mm}, k=0.17 \mathrm{~mm}$ ). Numbers 1-6 refer to compartments with different conductivities (Table I).

Intrafascicular conductivity was considered to be anisotropic [16]. All other compartments were assumed to be isotropic. The connective tissue surrounding the fascicles is rather inhomogeneous [11]. Therefore, we divided the extrafascicular connective tissue into two compartments: the perineural sheath enfolding the fascicles, and the epineural connective tissue which comprises all fascicles. Conductivities of these tissues could not be obtained from literature. The epineurium has a loose structure and partly consists of fat [11]. Therefore, its conductivity was assumed to be somewhat higher than the conductivity of fat tissue $\left(0.04(\Omega \mathrm{m})^{-1}[11]\right)$. The conductivity of the perineurium was assumed to be lower. During our experiments on rat the CP nerve was lifted from the surrounding tissue and embedded in parafin oil having a low conductivity. Therefore we assumed an extraneural conductivity of $0.01(\Omega \mathrm{m})^{-1}$ in the model of rat $\mathrm{CP}$ nerve, which is low compared to the epineural conductivity. Like Stegeman [17] did for human sural nerve, we assumed the extraneural conductivity of the human DP

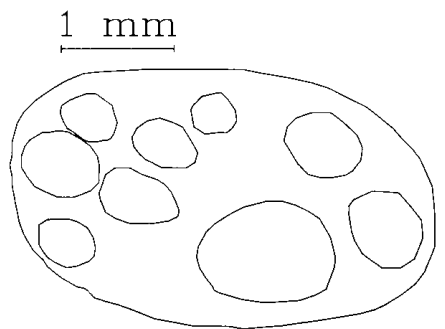

(a)

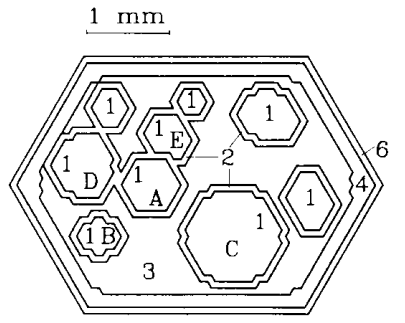

(b)

Fig. 3. Human deep peroneal (DP) nerve. (a) Cross-sectional anatomy at $24 \mathrm{~mm}$ below neck of fibula (after Sunderland [11]). (b) Cross section of nerve model ( $h=0.09 \mathrm{~mm}, k=0.21 \mathrm{~mm}$ ). Five fascicles were identified by $A \sim E$. For numbers $1-6$ see Table I.

TABLE I

Conductivity Values of the Media in the Compartments of the NERVE MODELS

\begin{tabular}{l|l|c|c|c|}
\hline \hline Model compartment & tissue & \multicolumn{3}{|c|}{ conductivity $(\Omega \mathrm{m})^{-1}$} \\
& & $\sigma_{\mathbf{x}}$ & $\sigma_{\mathbf{y}}$ & $\sigma_{\mathbf{z}}$ \\
\hline 1 & & 0.08 & 0.08 & 0.50 \\
2 & fascicle & 0.01 & 0.01 & 0.01 \\
3 & perineurium & 0.10 & 0.10 & 0.10 \\
4 (rat CP nerve) & epineurium & 0.01 & 0.01 & 0.01 \\
4 (human DP nerve) & fat & 0.04 & 0.04 & 0.04 \\
5 & blood vessel & 0.60 & 0.60 & 0.60 \\
6 & & $10^{-4}$ & $10^{-4}$ & $10^{-4}$ \\
\hline
\end{tabular}

nerve to have the same value as fat tissue. The volume elements at the surface of the model (compartment 6) were attributed a very low conductivity and are a compressed representation of the tissue at a larger distance from the nerve.

\section{Modeling of Nerve Fiber Excitation}

The relationship between stimulation field and membrane potential of a myelinated nerve fiber was modeled by the network description introduced by McNeal [18]. This results in a number of coupled differential equations, each one describing the potential at a single node of Ranvier:

$$
\begin{aligned}
\frac{d \varphi_{n}}{d t}= & \frac{1}{C_{m}}\left[G _ { a } \left[\left(\varphi_{n-1}-2 \varphi_{n}+\varphi_{n+1}\right)\right.\right. \\
& \left.\left.+\left(\varphi_{e, n-1}-2 \varphi_{e, n}+\varphi_{e, n+1}\right)\right]-G_{m \cdot \varphi n}\right]
\end{aligned}
$$


$\varphi_{n}$ is the deviation of the potential difference across the membrane from its resting value at node $n, C_{m}$ is the nodal membrane capacity and $G_{m}$ the nodal membrane conductance. $G_{a}$ is the intracellular conductance between two neighboring nodes. Like McNeal [18] we assumed that a linear relationship exists between the diameter $D$ and the internodal length $L$ of a nerve fiber ( $L=100 \mathrm{D}$ ) [18], [19], and that the part of the nerve fiber under consideration has a constant diameter. In the study of McNeal [18] the position of the node lying closest to the electrode (node 0 ), was confined to the cross-sectional plane of the electrode. Like McNeal et al. [20], [21] and Veltink et al. [22] we extended this model by varying the position of node $0 . L_{0}$ was defined as the distance in $z$ direction from the electrode to node 0 [22]

Like McNeal we used a nerve fiber model comprising 11 nodes of Ranvier numbered from -5 to 5 . For this number of nodes, the influence of the limited cable length is negligible. Node 0 was defined nearest to the stimulating electrode. The term $\varphi_{e, n-1}-2 \varphi_{e, n}+\varphi_{e, n+1}$ is the input term of the equations which was called the activating function by Rattay [23]. The threshold stimulation amplitude (TSA) of a nerve fiber is the stimulus pulse amplitude which depolarizes the nerve fiber membrane up to its excitation threshold. The number of nodes at which the activating function has to be considered for an accurate calculation of TSA depends on the potential distribution and the internodal length $L$. This number of nodes, which determines the length of the nerve model to be considered, was minimized in order to increase the resolution of the grid in $z$ direction (minimize $k$ ). In our simulations, we considered nerve fibers with $L=1.0 \mathrm{~mm}$ and $L=1.5$ $\mathrm{mm}$. In order to estimate TSA with an accuracy of 1 percent of its value, the activating function was calculated at nine nodes in all cases, except for $L=1.5 \mathrm{~mm}$ in the model of the small rat CP nerve (seven nodes). These numbers of nodes were empirically found by comparing the calculated TSA values for several numbers of nodes in a test with a larger extent of the model in $z$ direction (larger $k$ ). The activating function at the other peripheral nodes was set at zero. The rat CP nerve model had a length of $6.75 \mathrm{~mm}$. For the larger human DP nerve this length was $8.25 \mathrm{~mm}$. Consequently, the height $k$ of the volume elements was 0.17 and $0.21 \mathrm{~mm}$, respectively.

Monopolar cathodic stimulation was applied, with a pulse width of $60 \mu \mathrm{s}$, which is the same as we used in our stimulation experiments on rat CP nerve [24]. With increasing pulse amplitude $\varphi_{A}$ excitation will first occur at the node nearest to the electrode. The mechanism of excitation was modeled by the Frankenhaeuser-Huxley (FH) description of the membrane conductance $G_{m}[25]$ at three nodes of Ranvier. The membrane conductance of the other nodes was kept constant.

For each nerve fiber the distance $L_{0}$, the diameter $D$ and the position in the nerve cross section $\bar{r}(x, y)$ will influence TSA. These parameters have been described as stochastic variables by Veltink et al. [22], and the probability distributions of these variables have been used for the evaluation of nerve fiber excitation. In the present paper, nerve fiber excitation was evaluated for fixed values of diameter $D$ and distance $L_{0}$ [21]. Two nerve fiber diameters were considered $(10$ and $15 \mu \mathrm{m})$ and two extreme values of $L_{0}\left(L_{0}=0\right.$ and $\left.L_{0}=L / 2\right)$. Contour lines were determined in the nerve cross section. These contours surround the area in which nerve fibers, lying only in the fascicles (compartment 1), are predicted to be excited. For fixed diameter $D$ and unknown $L_{0}$ all nerve fibers will be excited inside the inner contour $\left(L_{0}=L / 2\right)$ and none will be excited outside the outer contour $\left(L_{0}=0\right)$. In the area between both contours nerve fiber excitation depends on $L_{0}$.

\section{RESULTS}

\section{Computational Test of the Volume Conduction Model}

To test the correctness of the computations, the electrical potential and the activating function in a simple concentric two cylinder nerve model were computed in two ways: using the analytical description by Veltink et al. [22], and the numerical method described in this paper. An intrafascicular electrode was located at the axis of the cylinder and was represented by a current point source in the analytical description. In the numerical method, an electrode with finite dimensions was represented by a Dirichlet boundary condition. The volume conduction parameters used were the same as in Veltink et al. [22]. For a suitably chosen ratio between the current through the electrode in the analytical description and the electrode potential in the numerical method, the field potentials and values of the activating function, compared at several positions, appeared to correspond well: they differed less than 5 percent of their value.

As to the convergence criterion (2) used in the numerical method described in this paper, it was found that 130 200 full model iterations were needed to meet the criterion, depending on the model geometry and the ratios of the conductivities. The criterion is a measure for the rate of convergence, not for the convergence itself. It can be computed much faster than the residual or the exact error and appeared to perform well in practice: it was tested that after meeting this criterion the solution in an example of human DP nerve did not change significantly when iterating an additional 1000 times. Convergence to the right solution was demonstrated in the two-cylinder model presented above.

\section{Nerve Fiber Recruitment in Rat CP Nerve}

Nerve stimulation by an extraneural and an intrafascicular electrode were simulated. The electrode positions corresponded with those in experiments on rat by Veltink et al. [8], [9]. The extraneural electrodes used experimentally had a diameter of $200 \mu \mathrm{m}$ and the diameter of the intrafascicular electrodes was $25 \mu \mathrm{m}$. The electrode surface can be considered as a circular disc, because the electrodes were insulated except at the very tip. In the model, the electrodes were represented by volume ele- 


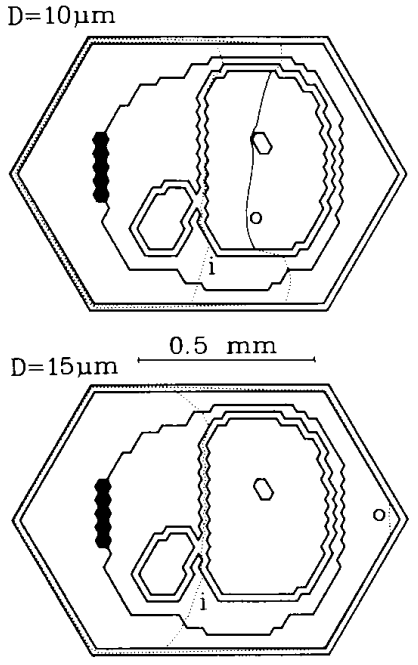

(a)

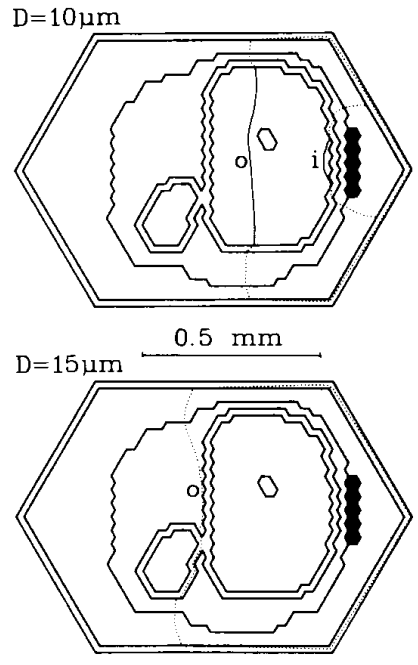

(b)
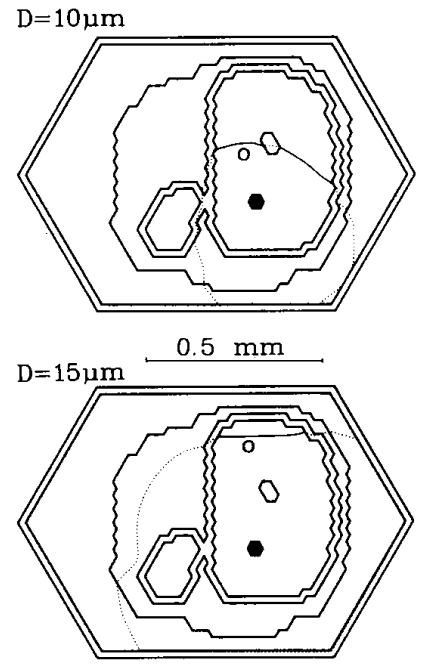

(c)

Fig. 4. Simulation of the recruitment of nerve fibers with diameters of 10 and $15 \mu \mathrm{m}$ in rat common peroneal nerve (contour $i: L_{0}=L / 2$ and contour o: $\left.L_{0}=0\right)$. (a) Stimulation on extraneural electrode $1\left(\varphi_{A}=\right.$

$-0.40 \mathrm{~V})$. (b) Stimulation on extraneural electrode $2\left(\varphi_{A}=-0.38 \mathrm{~V}\right)$.

(c) Stimulation on the intrafascicular electrode $\left(\varphi_{A}=-0.20 \mathrm{~V}\right)$.

ments. The diameter of the intrafascicular electrode was somewhat larger than in the experiments $(45 \mu \mathrm{m})$. Its height equals the height $k$ of one layer of the model $(0.17$ $\mathrm{mm})$.

For both extraneural and intrafascicular electrodes, contours for $L_{0}=0$ and $L_{0}=L / 2$ and fiber diameters of 10 and $15 \mu \mathrm{m}$ are shown in Fig. 4. At each electrode position a single stimulation pulse amplitude $\varphi_{A}$ was used. In Fig. 4(b) $(D=15 \mu \mathrm{m})$ and (c) $(D=10$ and $15 \mu \mathrm{m})$ the inner contours are absent, because no nerve fibers with $L_{0}=L / 2$ are excited. The results show that the contour lines of $L_{0}=0$ and $L_{0}=L / 2$ are relatively far apart, especially at larger internodal length $(D=15 \mu \mathrm{m})$. Thus, in the fascicle of rat CP nerve, TSA depends on the distance $L_{0}$ as well as on the position in the $x y$ plane.

TSA ranges of all nerve fibers with diameters $D=10$ $\mu \mathrm{m}$ and $D=15 \mu \mathrm{m}$ for $L_{0}=0$ and $L_{0}=L / 2$ in the main fascicle are shown in Fig. 5. These ranges represent the shift of the contour lines as a function of $\varphi_{A}$. At the lowest value of $\varphi_{A}$ the contour is just inside the fascicle, while at the highest value the contour comprises the whole fascicle. It can be seen that the recruitment ranges of fibers with $D=10 \mu \mathrm{m}$ and $D=15 \mu \mathrm{m}$ largely overlap. Therefore, no clear order of recruitment as a function of nerve fiber diameter can be concluded from these results. This corresponds to the finding in our experiments that mixed recruitment of motor units with different force contributions occurs, especially when using intrafascicular electrodes [8], [9]. As shown in Fig. 5, TSA can be lower for $10 \mu \mathrm{m}$ diameter fibers than for $15 \mu \mathrm{m}$ diameter fibers at $L_{0}=L / 2$, because the activating function for node 0 decreases sharply when $L_{0}$ increases and $L / 2$ is higher for $15 \mu \mathrm{m}$ than for $10 \mu \mathrm{m}$ diameter fibers. This phenomenon has been discussed by Veltink et al. [22].

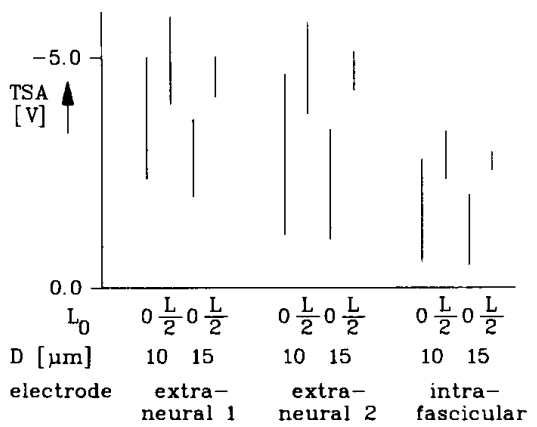

Fig. 5. Ranges in which the TSA varies between all nerve fibers in the main fascicle of rat CP nerve with $D=10 \mu \mathrm{m}$ and $D=15 \mu \mathrm{m}$ and for $L_{0}=0$ and $L_{0}=L / 2$. The TSA ranges are given for extraneural electrodes 1 and 2 and the intrafascicular electrode.

\section{Nerve Fiber Recruitment in Human DP Nerve}

Selective stimulation of fascicle $\boldsymbol{A}$ [Fig. 3(b)] inside this nerve was investigated by modeling an electrode outside the nerve, in the epineurium just outside the fascicle and inside this fascicle. Fascicle $A$ is situated in the middle of the nerve in between other fascicles. Recruitment contours for $L_{0}=0$ and $L_{0}=L / 2$ are shown in Fig. 6 for all three electrode positions and nerve fiber diameters 10 and $15 \mu \mathrm{m}$. Distances between the contours for $L_{0}=0$ and $L_{0}=L / 2$ appear to decrease at increasing distance from the electrode.

Fig. 7 shows TSA ranges of nerve fibers within each of the five fascicles $A-E$. Selective stimulation of fascicle $A$ in the middle of the nerve was best with the intrafascicular electrode and worst with the extraneural electrode. However, with an extraneural electrode selective stimulation of superficial fascicles near the electrode seems to be possible [see fascicle $B$ in Fig. 7(a)]. When the distance be- 

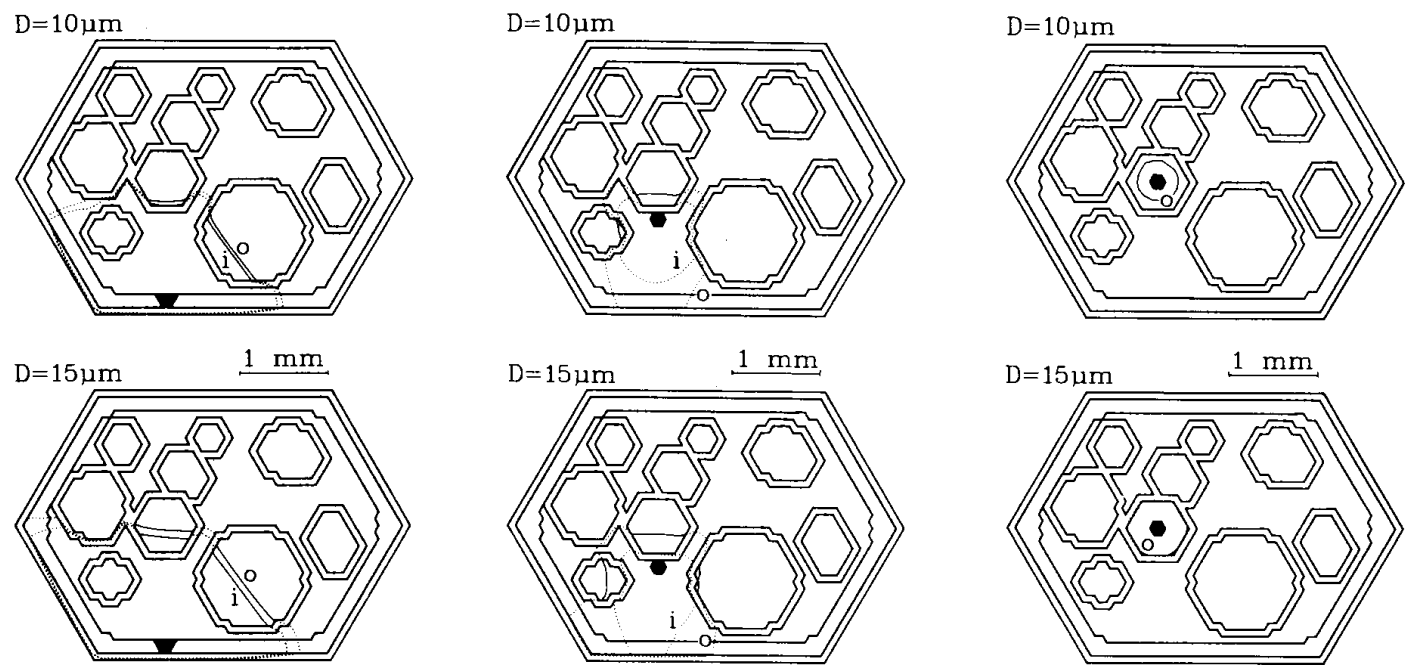

(b)

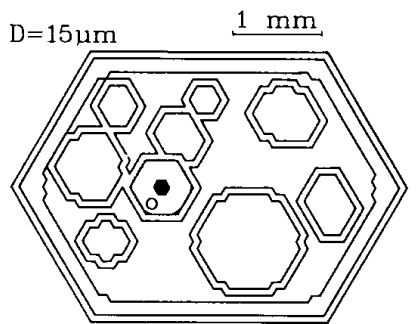

(c)

Fig. 6. Simulated nerve fiber recruitment in human deep peroneal nerve ( contour $i: L_{0}=L / 2$ and contour o: $L_{0}=0$ ). (a) Extraneural electrode $\left(\varphi_{A}=-1.87 \mathrm{~V}\right)$. (b) Epineural electrode just outside fascicle $\left(\varphi_{A}=\right.$ $-0.59 \mathrm{~V})$. (c) Intrafascicular electrode $\left(\varphi_{A}=-0.17 \mathrm{~V}\right)$.

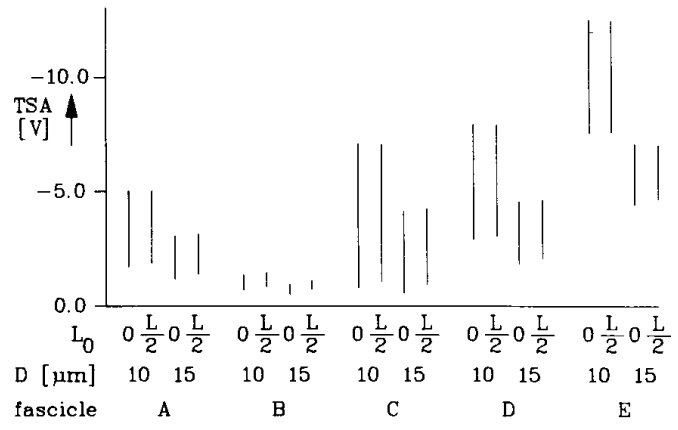

(a)

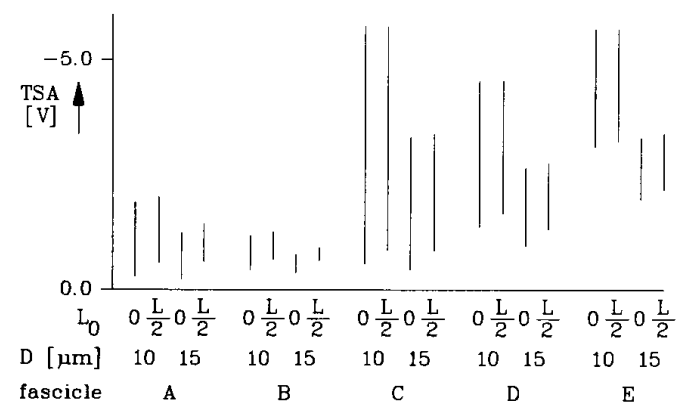

(b)

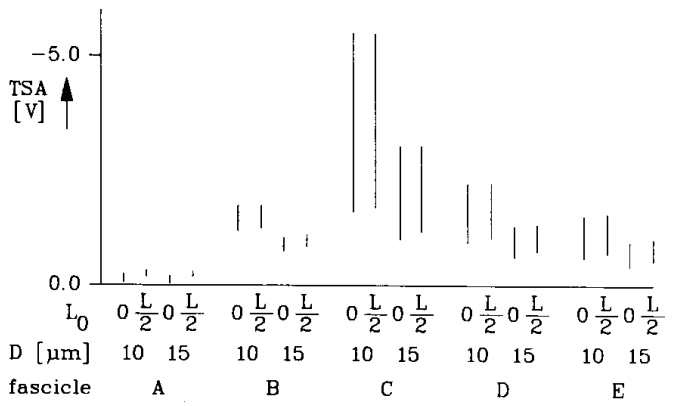

(c)

Fig. 7. Ranges in which the TSA varies between all nerve fibers lying in the fascicles $A-E$ of human DP nerve, for $D=10 \mu \mathrm{m}$ and $D=15 \mu \mathrm{m}$ and $L_{0}=0$ and $L_{0}=L / 2$. Same electrode positions as in Fig. 6. (a) Extraneural electrode. (b) Epineural electrode just outside fascicle. (c) Intrafascicular electrode.

tween electrode and recruited nerve fibers increases, the difference in TSA for different nerve fiber diameters increases. This can be seen by comparing TSA ranges of nerve fibers in fascicle $A$ between the extraneural electrode [Fig. 7(a)] and the intrafascicular electrode [Fig. 7(c)]. Fig. 7(a) furthermore shows that with an extra- 

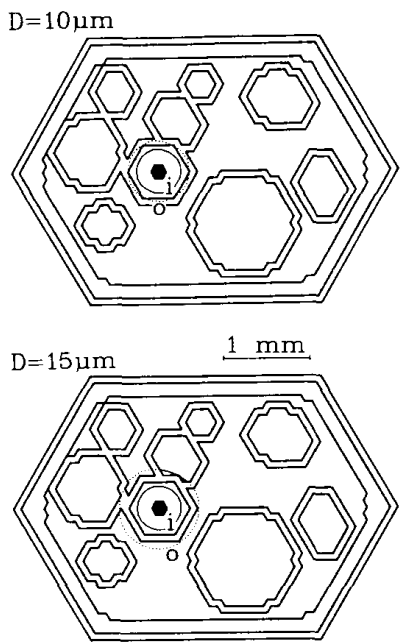

Fig. 8. Simulated nerve fiber recruitment in human deep peroneal nerve with conductivity of the perineurium identical to the conductivity of the epineurium $\left(0.1(\Omega \mathrm{m})^{-1}\right)$ for an intrafascicular electrode in fascicle $A$ $\left(\varphi_{A}=-0.35 \mathrm{~V}\right)$ (contour $i: L_{0}=L / 2$ and contour $\left.o: L_{0}=0\right)$.

neural electrode parts of fascicles closest to the electrode are recruited, as shown experimentally by McNeal and Bowman [6].

The influence of the perineural sheath conductivity on nerve fiber recruitment was investigated by executing the same simulations with a perineural sheath having the same conductivity as the epineurium $\left(0.1(\Omega \mathrm{m})^{-1}\right)$. An example of recruitment contours for intrafascicular electrodes is shown in Fig. 8. The low conductivity of the perineural sheath improves fascicle selectivity when using intrafascicular electrodes: As shown in Fig. 7(c) recruitment of nerve fibers of $15 \mu \mathrm{m}$ diameter was limited to the fascicle in which the electrode was placed, even when all nerve fibers in this fascicle were recruited. This was not found when assuming the perineural conductivity to be the same as the conductivity of the epineurium, even with partial recruitment of nerve fibers inside the fascicle (Fig. 8). Furthermore, the model predicted the influence of the conductance of the perineurium on fascicle selectivity to be less for epineural and extraneural stimulation (not shown in the figures).

\section{Discussion}

The numerical method presented for the computation of the potential distribution in a volume conductor of variable geometry enabled the modeling of nerve fiber excitation for a realistic anatomy and geometry of nerve and electrodes, rather than a simplified symmetrical geometry [22]. Furthermore, this numerical method enabled the incorporation of anisotropic media, in contrast to what is generally possible with other methods, for instance those based on Green's theorem [13].

The conductivity ratio of the media will influence recruitment characteristics [24]. For example, increasing the conductivity of the perineurium by a factor 10 to the value of the epineurium resulted in a worse fascicle selectivity for intrafascicular stimulation (Figs. 6(c) and 8). However, the results in Figs. 4-7 show that the positions of electrode and fascicles in the nerve cross section and the stimulus parameters (e.g., stimulus amplitude) are main determinants of the recruitment characteristics.

The conductivity of compartment 6 will not influence the potential distribution in the rat $\mathrm{CP}$ nerve model in a significant way because of the low conductivity of compartment 4 . Whether in the case of human DP nerve, especially for the extraneural electrode, a low conductivity of compartment 6 is a sufficient compressed representation of the tissue at a larger distance from the nerve could be investigated by modeling a much larger extraneural compartment with the same conductivity as compartment 4. However, varying grid resolution would be necessary in order to ensure a sufficient grid resolution in the nerve, while modeling a large extraneural space with a limited number of grid points.

The McNeal model of nerve fiber excitation is based on a number of assumptions:

- The simultaneous excitation of many nerve fibers during a stimulation pulse was assumed not to influence the potential distribution in the macroscopic volume conductor.

- The myelin sheath surrounding the nerve fibers between subsequent nodes was assumed to be a perfect isolator.

- Blocking of action potential propagation along nerve fibers lying close to the cathodal stimulating electrode was neglected. This blocking could occur because of hyperpolarization at nodes of Ranvier at some distance along the nerve fibers [26]. Blocking occurs at much larger stimulus amplitudes than the threshold amplitude for excitation: Ranck [26] mentions a factor 8 between excitation and blocking threshold. However, this factor must be expected to increase for decreasing stimulus width [7], and to be influenced by the distance between electrode and nerve fiber.

- The description of the membrane characteristics was based on measurements on frogs [25] rather than on mammals. Sweeney et al. [27] introduced a new set of equations for modeling the membrane conductance of myelinated nerve fibers. This description would be more adequate for human nerve fibers at $37^{\circ} \mathrm{C}$ than the Frankenhaeuser-Huxley equations, which we used for compatibility with our former simulation model [22]. We compared the influence of both models of the membrane conductance in the case of epineural stimulation of human DP nerve by calculating recruitment contours. The main difference was a lower excitation threshold when using the Sweeney equations. Recruitment characteristics were also influenced to some extent, but do not lead to other conclusions than presented in this paper.

As described, the model predictions appeared to correspond reasonably well with experimental results [6], [8], [9], [28], despite the assumptions presented above. 
The large influence of nodal positions in the $z$ direction on TSA in simulations of rat CP nerve indicates a large spread of the motoneuron group which is recruited by one electrode. This contributes to an extensive overlap within one fascicle of motoneuron groups recruited by different electrodes, placed in or near to the fascicle. This was found in our experiments with extraneural and intrafascicular multielectrode stimulation of rat CP nerve [8], [9]. The model also predicts a rather mixed recruitment of large and small diameter nerve fibers at any submaximal stimulation level. This was also observed in experiments by Veltink et al. [8], [9] for intrafascicular electrodes.

For larger distances between electrode and nerve fibers, the influence of node positions on TSA will decrease, and a more pronounced inverse order of recruitment with respect to nerve fiber diameter will occur. This means that a higher percentage of large diameter nerve fibers are present in the recruited population of motoneurons at a given stimulus level. Pronounced inverse recruitment was found experimentally for extraneural stimulation by Petrofsky [3] and Fang and Mortimer [7].

The model results indicate that muscle selective stimulation with extraneural electrodes will be possible when muscle specificity differs among fascicles at different sides of a nerve. This corresponds to the results of the muscle selective stimulation experiments by $\mathrm{McNeal}$ and Bowman [6].

The model presented predicts that selective stimulation of a fascicle by an intrafascicular electrode will be possible. Additional stimulation of parts of neighboring fascicles may occur when stimulating by an electrode in the epineurium just outside a fascicle. Fascicle selective stimulation with epineural and intrafascicular electrodes should be investigated experimentally, because it may improve the performance of multielectrode nerve stimula-

$$
\text { plane (equilateral triangle) }
$$

$\varphi(x, y, z) \quad$ Electrical potential at point $(x, y, z)$

$\varphi_{i}^{j} \quad$ Electrical potential at grid point $i$, after iteration $j$

$V \quad$ Volume

$\sigma_{x}, \sigma_{y}, \sigma_{z} \quad$ Electrical conductivities in $x, y$, and $z \mathrm{di}$ rections

$D \quad$ Nerve fiber diameter

$L \quad$ Internodal length ( $L=100 \mathrm{D}$ [18], [19])

$L_{0} \quad$ Distance in $z$ direction from the electrode to node 0 .

$C_{m} \quad$ Nerve fiber nodal membrane capacity

$G_{m} \quad$ Nerve fiber nodal membrane conductance

$G_{a} \quad$ Intracellular conductance between two neighboring nodes

$\varphi_{e, n} \quad$ Extracellular potential at node $n$

$\varphi_{n} \quad$ Potential across nerve fiber membrane at node $n$ minus its resting value

TSA Threshold stimulation amplitude

$\varphi_{A} \quad$ Stimulus pulse amplitude

\section{APPENDIX B}

For the computation of the electrical potential distribution, the dissipation $D_{e}(\varphi)$ was calculated for every volume element. $D_{e}(\varphi)$ was defined as a function of the potentials at the six corner points of a volume element:

$$
D_{e}(\bar{\varphi})=\bar{\varphi}^{T} \cdot M_{e} \cdot \bar{\varphi}
$$

$\bar{\varphi}^{T}=\left(\varphi_{1}, \varphi_{2}, \varphi_{3}, \varphi_{4}, \varphi_{5}, \varphi_{6}\right)$ is the vector of the electrical potentials at the six corner points of the volume element (figure 1). Matrix $\boldsymbol{M}_{e}$ was defined as

$$
\boldsymbol{M}_{e}=\frac{1}{12 \sqrt{3}}\left[\begin{array}{cccccc}
3 s_{x}+s_{y}+2 s_{z} & -3 s_{x}+s_{y} & -2 s_{y} & -2 s_{z} & 0 & 0 \\
-3 s_{x}+s_{y} & 3 s_{x}+s_{y}+2 s_{z} & -2 s_{y} & 0 & -2 s_{z} & 0 \\
-2 s_{y} & -2 s_{y} & 4 s_{y}+2 s_{z} & 0 & 0 & -2 s_{z} \\
-2 s_{z} & 0 & 0 & 3 s_{x}+s_{y}+2 s_{z} & -3 s_{x}+s_{y} & -2 s_{y} \\
0 & -2 s_{z} & 0 & -3 s_{x}+s_{y} & 3 s_{x}+s_{y}+2 s_{z} & -2 s_{y} \\
0 & 0 & -2 s_{z} & -2 s_{y} & -2 s_{y} & 4 s_{y}+2 s_{z}
\end{array}\right]
$$

tion systems. Although stimulation with intraneural or intrafascicular electrodes for long term nerve stimulation needs further investigation, results of chronic implantation are promising [29].

\section{Appendix A}

\section{List of Symbols}

$\begin{array}{ll}D(\varphi) & \text { Dissipation functional } \\ D_{e}(\varphi) & \text { Dissipation functional of a volume element }\end{array}$
With

$$
\begin{aligned}
& s_{x}=\sigma_{x} k \\
& s_{y}=\sigma_{y} k \\
& s_{z}=\sigma_{z} \frac{h^{2}}{k} .
\end{aligned}
$$

Matrix $\boldsymbol{M}$ was derived by first writing the dissipation in a volume element according to formula (1), limiting the in- 
tegration to this volume element. Then a transformation was performed from the $x y z$ coordinate system to the $\xi \eta \zeta$ coordinate system (Fig. 1). Finally, the differentials in the integral expression were approximated by finite differences in the $\xi \eta \zeta$ coordinate system.

\section{ACKNOWLEDGMENT}

We would like to thank F. P. H. van Beckum from the Department of Applied Mathematics, University of Twente, for helpful discussions and advice. Furthermore, we would like to thank P. Wirtz and H. M. T. Loermans of the University of Nijmegen, The Netherlands, for the histochemical preparation of rat common peroneal nerve.

\section{REFERENCES}

[1] J. Holle, E. Moritz, and H. Thoma, "Die Karusselstimulation, eine neue Methode zur elektrophrenische Langzeitbeatmung," Wiener klinische Wochenschrift, vol. 86, pp. 23-27, 1974.

[2] J. S. Petrofsky, "Sequential motor unit stimulation through peripheral motor nerves in the cat," Med. Biol. Eng. Comput., vol. 17, pp. 87-93, 1979.

[3] J. S. Petrofsky and C. A. Phillips, "Impact of recruitment order on electrode design for neural prosthetics of skeletal muscle," Amer. J. Phys. Med., vol. 60, pp. 243-253, 1981.

[4] M. Solomonow, E. Eldred, J. Lyman, and J. Foster, "Control of muscle contractile force through indirect high-frequency stimulation," Amer. J. Phys. Med., vol. 62, pp. 71-82, 1983.

[5] M. Solomonow, "External control of the neuromuscular system," IEEE Trans. Biomed. Eng., vol. BME-31, pp. 752-763, 1984.

[6] D. R. McNeal and B. R. Bowman, "Selective activation of muscles using peripheral nerve electrodes," Med. Biol. Eng. Comput., vol. 23, pp. 249-253, 1985.

[7] Z.-P. Fang and J. T. Mortimer, "A method for attaining natural recruitment order in artificially activated muscles," in Proc. 9th Annu. Conf. IEEE Eng. Med. Biol. Soc., Boston, MA, 1987, pp. 657-658.

[8] P. H. Veltink, H. J. Hermens, and J. A. van Alsté, "Multielectrode intrafascicular and extraneural Stimulation," in Proc. 8th Annu. Conf. IEEE Eng. Med. Biol. Soc., Dallas, TX, 1986, pp. 690-693.

[9] P. H. Veltink, J. A. van Alsté, and H. B. K. Boom, "Multielectrode intrafascicular and extraneural stimulation," Med. Biol. Eng. Comput., vol. 27, pp. 19-24, 1989.

[10] E. Moritz, J. Holle, H. Thoma, and H. Stöhr, "Further experiences with elektrophrenic respiration," in Proc. Ist Vienna Internat. Work shop Functional Electrostimulation, 1983.

[11] S. Sunderland, Nerves and Nerve Injuries. Edinburgh and London: Livingstone, 1968.

[12] Y. Yamashita and T. Takahashi, "Use of the finite element method to determine epicardial from body surface potentials under a realistic torso model," IEEE Trans. Biomed. Eng., vol. BME-31, pp. 611$621,1984$.

[13] T. C. Pilkington, M. N. Morrow, and P. C. Stanley, "A comparison of finite element and integral equation formulations for the calculation of electrocardiographic potentials," IEEE Trans. Biomed. Eng., vol. BME-32, 166-173, 1985

[14] G. Dahlquist and Ö. Björck, Numerical Methods. Englewood Cliffs, NJ: Prentice Hall, 1974.

[15] W. Krücke, "Pathologie der peripheren Nerven,"' in Handbuch der Neurochirurgie, Peripheres und Sympathisches Nervensystem. H. Olivecrona, W. Tönnis, and W. Krenkel, Eds. New York: Springer, 1974, pp. 1-142.

[16] L. A. Geddes and L. E. Baker, "The specific resistance of biological material-A compendium of data for the biomedical engineer and physiologist," Med. Biol. Eng., vol. 5, pp. 271-293, 1967.

[17] D. F. Stegeman and J. P. C. de Weerd, "Modeling compound action potentials of peripheral nerves in situ. I. Model description; evidence for a nonlinear relation between fiber diameter and velocity," Electroencephalogr. clin. Neurophysiol., vol. 54, pp. 436-448, 1982.

[18] D. R. McNeal, "Analysis of a model for excitation of myelinated nerve,"' IEEE Trans. Biomed. Eng., vol. BME-23, pp. 329-337, 1976.

[19] P. K. Thomas and J. Ochoa, "Microscopic anatomy of peripheral nerve fibers," in Peripheral Neuropathy, Vol. 1, P. J. Dyck, P. K. Thomas, E. H. Lambert, and R. Bunge, Eds. Philadelphia: Saunders, 1984, pp. 39-96.

[20] D. R. McNeal and D. A. Teicher, "Effect of electrode placement on threshold and initial site of excitation of a myelinated nerve fiber." in Functional Electrical Stimulation, F. T. Hambrecht and J. B. Reswick, Eds. New York: Marcel Dekker 1977, pp. 405-412.

[21] D. R. McNeal and A. V. Apkarian, "Analytical design of a cuff electrode," in Proc. 6th Int. Symp. External Contr. Hum an Extremities, Dubrovnik, pp. 45-54, 1978.

22] P. H. Veltink, J. A. van Alsté, and H. B. K. Boom, "Simulation of intrafascicular and extraneural nerve stimulation," IEEE Trans. Biomed. Eng., vol. 35, 69-75, 1988.

[23] F. Rattay, "Analysis of models for external stimulation of axons," IEEE Trans. Biomed. Eng., vol. BME-33, pp. 974-977, 1986.

[24] P. H. Veltink, J. A. van Alsté and H. B. K. Boom, "Influences of stimulation conditions on recruitment of myelinated nerve fibers: A model study," IEEE Trans. Biomed. Eng., vol. 35, pp. 917-924, 1988.

[25] B. H. Frankenhaeuser and A. F. Huxley, "The action potential in the myelinated nerve fiber of Xenopus Laevis as computed on the basis of voltage clamp data,' J. Physiol., vol. 171, pp. 302-315, 1964.

[26] J. B. Ranck, "Which elements are excited in electrical stimulation of mammalian central nervous system: A review," Brain Res., vol. 98 , pp. $417-440,1975$.

[27] J. D. Sweeney, J. T. Mortimer, and D. Durand, "Modeling of mammalian myelinated nerve for functional neuromuscular stimulation." in Proc. 9th Annu. Conf. IEEE Eng. Med. Biol. Soc., Boston, MA. 1987, pp. 1577-1578.

[28] P. H. Gorman and J. T. Mortimer, "The effect of stimulus parameters on the recruitment characteristics of direct nerve stimulation," IEEE Trans. Biomed. Eng., vol. BME-30, pp. 407-414, 1983

[29] B. R. Bowman and R. C. Erickson, "Acute and chronic implantation of coiled wire intraneural electrodes during cyclical electrical stimulation," Ann. Biomed. Eng., vol. 13, pp. 75-93, 1985.

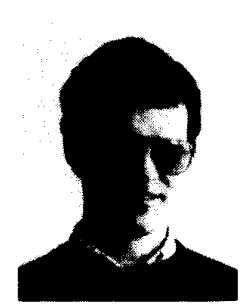

Peter H. Veltink (M'88) studied electrical engineering at the University of Twente, Enschede, The Netherlands, where he received the M.Sc. degree in 1984 and the $\mathrm{Ph} . \mathrm{D}$. degree in 1988 (dissertation: recruitment of myelinated nerve fibers during artificial nerve stimulation).

Currently, he is a member of the Biomedical Engineering Division of the Department of Electrical Engineering, University of Twente and is engaged in the research of neuromuscular stimulation for rehabilitation of spinal cord injured patients, which is carried out in cooperation with the Roessingh rehabilitation center in Enschede. His research interests are nerve stimulation and control systems for functional neuromuscular stimulation.

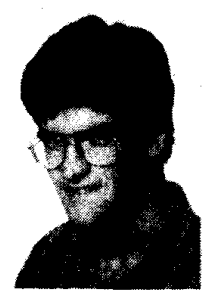

Benno K. van Veen was born in Vaassen, the Netherlands, in 1963. He received the M.Sc. de gree in electrical engineering from the University of Twente, Enschede, The Netherlands, in 1988

Since 1988 he has been working towards the Ph.D. degree at the Department of Biomedical Engineering, Faculty of Electrical Engineering at the University of Twente. His current research activities involve the electrical activity and volume conduction in skeletal muscle.

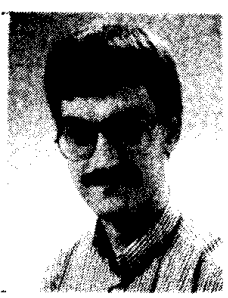

Johannes J. Struijk was born in Rijssen, The Netherlands, in 1963. He received the M.Sc. degree in electrical engineering in 1988 from the University of Twente where he currently is a $\mathrm{Ph} . \mathrm{D}$. student. His research interest is related to spinal cord stimulation, especially volume conduction and neural modeling. 


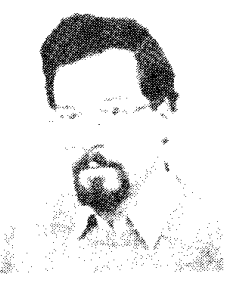

vous tissue.
Jan Holsheimer was born in Enschede, The Netherlands, in 1941 . He received the M.Sc. degree in biology and biophysics from the University of Groningen. The Netherlands, in 1965 and the $\mathrm{Ph} . \mathrm{D}$. degree in biomedical engineering from the University of Twente. Enschede, in 1982

In 1965 he joined the Biomedical Engineering Division, Department of Biomedical Engineering. University of Twente. His research interests are volume conduction and the analysis of field potentials in the brain and electrical stimulation of ner-

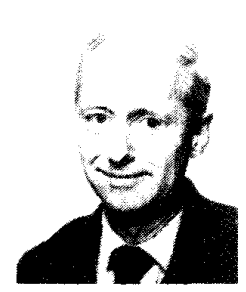

Herman B. K. Boom was trained as a Medical Physicist at the University of Utrecht, The Netherlands, where he received the Ph.D. degree in 1971.

He joined the Departments of Medical Physics and Medical Physiology where he was engaged in research in the field of cardiac mechanics and taught physiology and biophysics. Since 1976 he holds the Chair of Medical Electronics in the Department of Electrical Engineering. University of Twente, The Netherlands. His research interests are cardiovascular system dynamics, bioelectricity, and rehabilitation technology. 